\title{
Development of a plasmid stabilization system in Vibrio natriegens for the high production of 1,3-propanediol and 3-hydroxypropionate
}

\author{
Ye Zhang ${ }^{1}$, Qing Sun ${ }^{1}$, Yu Liu' ${ }^{1}$ Xuecong Cen ${ }^{1}$, Dehua Liu ${ }^{1,2,3}$ and Zhen Chen ${ }^{1,2,3^{*}}$ (D)
}

\begin{abstract}
Vibrio natriegens is a promising industrial chassis with a super-fast growth rate and high substrate uptake rates. $V$. natriegens was previously engineered to produce 1,3-propanediol (1,3-PDO) from glycerol by overexpressing the corresponding genes in a plasmid. However, antibiotic selection pressure for plasmid stability was not satisfactory and plasmid loss resulted in reduced productivity of the bioprocess. In this study, we developed an antibiotic-free plasmid stabilization system for $V$. natriegens. The system was achieved by shifting the glpD gene, one of the essential genes for glycerol degradation, from the chromosome to plasmid. With this system, engineered $V$. natriegens can stably maintain a large expression plasmid during the whole fed-batch fermentation and accumulated 69.5 g/L 1,3-PDO in $24 \mathrm{~h}$, which was $23 \%$ higher than that based on antibiotic selection system. This system was also applied to engineering $\mathrm{V}$. natriegens for the production of 3-hydroxypropionate (3-HP), enabling the engineered strain to accumulate $64.5 \mathrm{~g} / \mathrm{L}$ 3-HP in $24 \mathrm{~h}$, which was 30\% higher than that based on antibiotic system. Overall, the developed strategy could be useful for engineering $V$. natriegens as a platform for the production of value-added chemicals from glycerol.
\end{abstract}

Keywords: Vibrio natriegens, 1,3-propanediol, 3-hydroxypropionate, Plasmid maintenance, Antibiotic free, Glycerol

\footnotetext{
*Correspondence: zhenchen2013@mail.tsinghua.edu.cn

1 Key Laboratory of Industrial Biocatalysis (Ministry of Education),

Department of Chemical Engineering, Tsinghua University,

Beijing 100084, China

Full list of author information is available at the end of the article
} 


\section{Graphic Abstract}

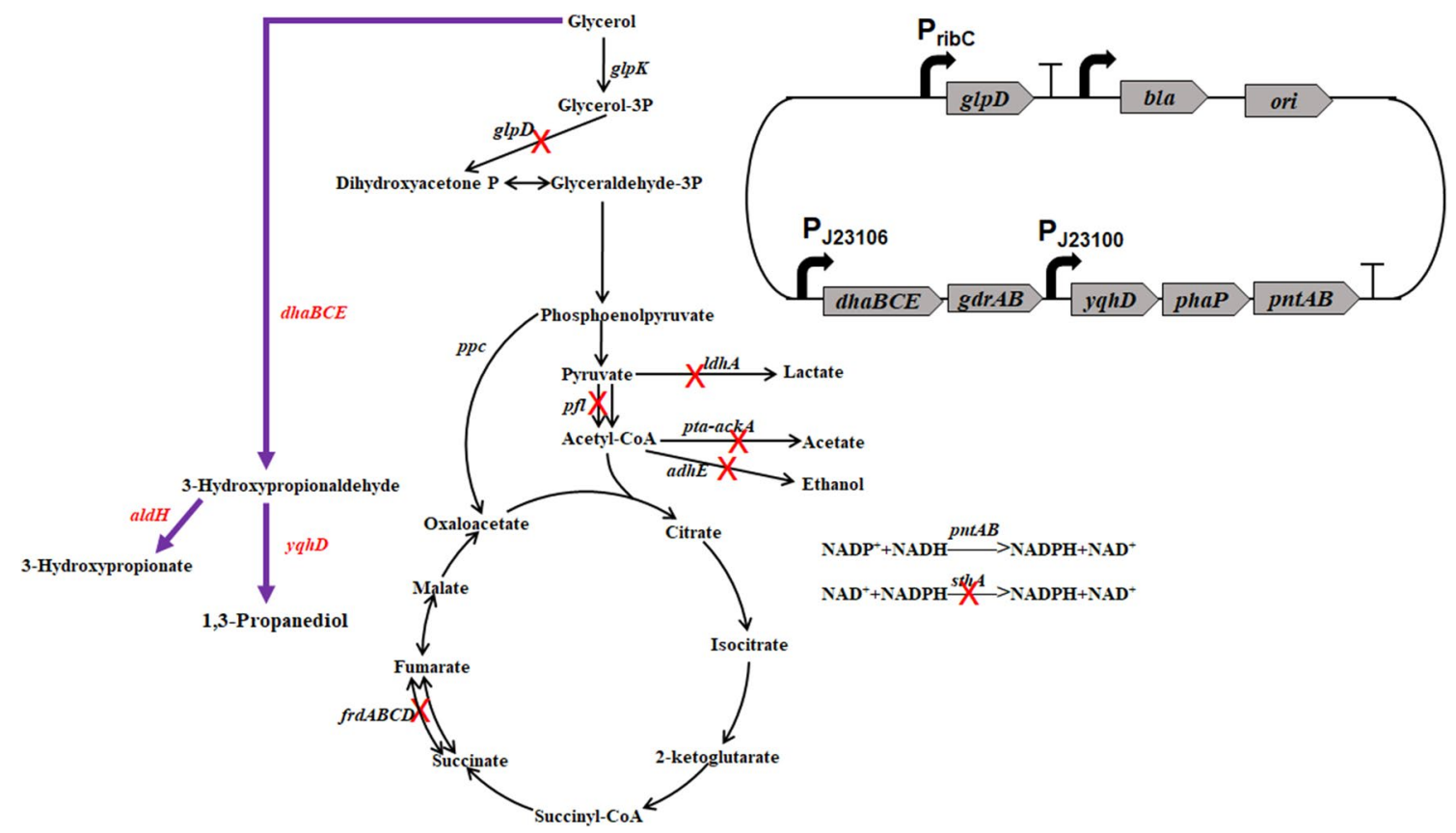

\section{Introduction}

The development of economically competitive bioprocesses to produce fuels and chemicals from renewable bioresources is important to achieve the goal of carbon neutrality (Becker and Wittmann 2015; Clomburg et al. 2017). In the past twenty years, metabolic engineering strategies have been widely used to improve the titers and yields of bioproducts, which have significantly increased the economic viability of bioprocesses (Kim et al. 2020; Ko et al. 2020). On the other hand, the productivity of a bioprocess is often limited by the maximum growth rate and/or substrate uptake rate of the employed microorganism (Zhang et al. 2017). Thus, developing fast-growing microorganisms as new industrial chassis could be important for improving the productivity of fermentation processes (Hoffart et al. 2017).

Vibrio natriegens is a promising next-generation chassis for industrial biotechnology which has a remarkably short doubling time of less than $10 \mathrm{~min}$ in complex media (Weinstock et al. 2016). It can utilize diverse substrates and has an exceptionally high glucose uptake rate under both aerobic and anaerobic conditions (Hoffart et al. 2017), making it an attractive candidate for industrial application. In recent years, different genetic tools and metabolic engineering strategies have been developed to engineer $V$. natriegens to produce value-added chemicals, including alanine, PHB, melanin, 2,3-butanediol, and 1,3-propanediol (Dalia et al. 2017; Hoffart et al. 2017; Lim et al. 2019; Wang et al. 2020; Zhang et al. 2021). In a previous study, we have developed a recombinant $V$. natriegens to efficiently produce 1,3-propanediol (1,3-PDO) from glycerol by introducing and balancing 1,3-PDO synthesis module in a plasmid and systematically optimizing glycerol metabolism and cofactor balance (Zhang et al. 2021). The engineered strain can produce $56.2 \mathrm{~g} / \mathrm{L} 1,3-$ PDO with a high productivity of $2.36 \mathrm{~g} / \mathrm{L} / \mathrm{h}$.

Plasmid-based expression systems have been widely used in industrial biotechnology for developing recombinant strains (Kang et al. 2018). Easy manipulation and high-level expression of targeted genes facilitate their wide application. However, the instability of plasmidbased expression systems may hinder their large-scale application in industry. Although antibiotics can be used during fermentation to reduce plasmid loss, the addition of antibiotics to large-scale cultures is expensive and the release of antibiotics to the environment is strongly disfavored (Terrinoni et al. 2018). Moreover, plasmid loss and strain heterogeneity are often observed in large-scale fermentation even with antibiotics addition due to fast cell growth and heterogeneous environments (Rugbjerg and Sommer 2019; Kang et al. 2018). Thus, the development of stable plasmid expression and maintenance systems without using antibiotics is important for industrial application.

Several antibiotic-free plasmid systems have been previously developed to stably maintain plasmids in 
Escherichia coli (Kang et al. 2018; Terrinoni et al. 2018). These systems are normally based on essential gene complementation systems or toxin-antitoxin systems. The implementation of these systems is often very complicated and the application of these systems for the high-level production of chemicals has not been demonstrated. In this study, we discovered that plasmid loss was a key factor affecting the production of 1,3-PDO from glycerol by previously designed $V$. natriegens. A plasmid maintenance system was developed by deleting the $g l p D$ gene encoding glycerol-3-phosphate dehydrogenase which is essential for glycerol metabolism and by complementing the gene in the expression plasmid. The system was proved to be efficient to increase the plasmid stability and productivity of 1,3-PDO. The system was also successfully applied to develop an efficient $V$. natriegens strain to produce 3-hydroxypropionate (3-HP) from glycerol. To the best of our knowledge, this is the first demonstration of 3-HP production by $V$. natriegens.

\section{Materials and methods}

Bacterial strains and plasmids

The bacterial strains and plasmids used in this study are listed in Table 1. Plasmids construction was based on the cloning host E. coli DH5 $\alpha$. V. natriegens VN09 was derived from the wild-type $V$. natriegens ATCC14048 by deleting ten genes related to byproducts formation and metabolic regulation ( $\triangle a d h E \Delta l d h A \Delta p f l \Delta p t a-a c k A \Delta \operatorname{arc} A$ $\Delta g l p R \triangle$ sthA $\triangle$ frdABCD $\triangle$ aldA $\triangle$ aldB) (Zhang et al. 2021). High-copy plasmid pTrc99a was used for gene overexpression in $V$. natriegens.

\section{Plasmids construction}

To construct plasmid pTrc99a-J23106-doy-phaP-pntABglpD1, the native promoter of $d n a G$ gene and the $\operatorname{glp} D$ gene were amplified from $V$. natriegens ATCC14048. The $\mathrm{T} 1$ terminator was amplified from plasmid pTrc99a. The three fragments were inserted into the XbaI site of pTrc99a-J23106-doy-phaP-pntAB-glpD1 (Zhang et al. 2021) by the standard protocol of Gibson assembly. The

Table 1 Strains and plasmids used in this study

\begin{tabular}{|c|c|c|}
\hline Strains or plasmids & Description & References \\
\hline \multicolumn{3}{|l|}{ Plasmids } \\
\hline pTrc99a & High-copy expression plasmid, Amp ${ }^{R}$ & Lab collection \\
\hline pTrc99a-J23106-doy-phaP-pntAB & $\begin{array}{l}\text { pTrc99a containing dhaBCE and gdrAB genes from K. pneumoniae under the control } \\
\text { of J } 23106 \text { promoter, an artificial operon containing yahD gene from E. coli, phaP } \\
\text { gene from Azotobacter sp., and pntAB genes from E. coli under the control of J } 23100 \\
\text { promoter }\end{array}$ & Zhang et al. (2021) \\
\hline pTrc99a-J23106-doy-phaP-pntAB-glpD1 & $\begin{array}{l}\text { pTrc99a-J23106-doy-phaP-pntAB containing glpD gene under the control of } V \text {. natrie- } \\
\text { gens'dnaG promoter }\end{array}$ & This study \\
\hline pTrc99a-J23106-doy-phaP-pntAB-glpD2 & $\begin{array}{l}\text { pTrc99a-J23106-doy-phaP-pntAB containing glpD gene under the control of } V \text {. natrie- } \\
\text { gens' patZ promoter }\end{array}$ & This study \\
\hline pTrc99a-J23106-doy-phaP-pntAB-glpD3 & $\begin{array}{l}\text { pTrc99a-J23106-doy-phaP-pntAB containing glpD gene under the control of } \mathrm{V} \text {. natrie- } \\
\text { gens' ribC promoter }\end{array}$ & This study \\
\hline pTrc99a-dhaBCE-aldH & $\begin{array}{l}\text { pTrc99a containing dhaBCE and gdrAB genes from K. pneumoniae under the control of } \\
\text { J23106 promoter, an artificial operon containing aldH gene from E. coli and phaP gene } \\
\text { from Azotobacter sp. under the control of } J 23100 \text { promoter }\end{array}$ & This study \\
\hline pTrc99a-dhaBCE-aldH-glpD3 & pTrc99a-dhaBCE-aldH containing glpD gene under the control of ribC promoter & This study \\
\hline \multicolumn{3}{|l|}{ Strains } \\
\hline V. natriegens ATCC14048 & Wild-type V. natriegens & Lab collection \\
\hline VN09 & $\begin{array}{l}\text { V. natriegens ATCC14048, } \triangle a d h E \triangle I d h A \triangle p f l \triangle p t a-a c k A \triangle a r c A \triangle g l p R \triangle s t h A \\
\triangle \text { frdABCD } \triangle a l d A \triangle a l d B\end{array}$ & Zhang et al. (2021) \\
\hline VN10 & VN9, harboring pTrc99a-J23106-doy-phaP-pntAB & Zhang et al. (2021) \\
\hline VN10 $\triangle \mathrm{glpD}$ & VN10, $\Delta g / p D$ & This study \\
\hline VN11 & VN09, $\Delta g l p D$, harboring pTrc99a-J23106-doy-phaP-pntAB-glpD1 & This study \\
\hline VN12 & VN09, $\triangle g l p D$, harboring pTrc99a-J23106-doy-phaP-pntAB-glpD2 & This study \\
\hline VN13 & VN09, $\triangle g l p D$, harboring pTrc99a-J23106-doy-phaP-pntAB-glpD3 & This study \\
\hline VN05 & V. natriegens ATCC14048, $\triangle a d h E \triangle I d h A \triangle p f l \triangle p t a-a c k A \triangle$ frdABCD & This study \\
\hline VN14 & VN05, harboring pTrc99a-dhaBCE-aldH & This study \\
\hline VN05 $\triangle \mathrm{glpD}$ & VN05, $\triangle g l p D$ & This study \\
\hline VN15 & VN05 $\Delta$ glpD, pTrc99a-dhaBCE-aldH-glpD3 & This study \\
\hline
\end{tabular}


"doy" within the plasmid name indicates the dhaBCE$g d r A B-y q h D$ gene cluster.

Plasmids pTrc99a-J23106-doy-phaP-pntAB-glpD2 and pTrc99a-J23106-doy-phaP-pntAB-glpD3 were constructed by replacing the $d n a G$ promoter of plasmid pTrc99a-J23106-doy-phaP-pntAB-glpD1 with the native promoter of pat $Z$ gene and $r i b C$ gene of $V$. natriegens ATCC14048 by Gibson assembly.

To construct plasmid pTrc99a-dhaBCE-aldH, the backbone of plasmid pTrc99a-J23106-doy-phaP-pntAB was amplified using PT-F and PT-R primers. The aldH gene was amplified from E. coli MG1655 using aldH-F and aldH-R primers. The phaP gene was amplified from pTrc99a-J23106-doy-phaP-pntAB using phaP-F and phaP-R primers. The three fragments were assembled by using Gibson assembly kits. To obtain plasmid pTrc99adhaBCE-aldH-glpD3, a fragment containing ribC promoter, $g l p D$ gene, and $\mathrm{T} 1$ promoter was amplified from plasmid pTrc99a-J23106-doy-phaP-pntAB-glpD3 and inserted into the XbaI site of pTrc99a-dhaBCE-aldH. All primers used in this study are listed in Additional file 1: Table S1.

\section{Strains construction}

To delete genes in $V$. natriegens genome, the standard protocol combining natural transformation and FLP/ FRT recombination system was used as described before (Zhang et al. 2021). A DNA fragment containing the upstream fragment ( $3000 \mathrm{bp})$ of the deleted gene, the resistance gene with two FRT loci, and the downstream fragment ( $3000 \mathrm{bp})$ of the deleted gene was obtained by overlap extension PCR. The recombinants with selection marker were obtained using natural transformation by following the protocols described by Dalia et al. (2014). To eliminate the selection marker, the selected strain was cultured in LBv2 medium with $1 \mathrm{mM}$ rhamnose at $37{ }^{\circ} \mathrm{C}$, $200 \mathrm{rpm}$ for $12 \mathrm{~h}$.

\section{Medium and culture conditions}

The LBv2 medium (LB broth supplemented with $200 \mathrm{mM}$ $\mathrm{NaCl}$, 23.14 $\mathrm{mM} \mathrm{MgCl}_{2}$, and $4.2 \mathrm{mM} \mathrm{KCl}$ ) is generally used for the culture of $V$. natriegens. Modified VN medium is used for the fermentation of $V$. natriegens to produce 1,3-PDO and 3-HP, containing $\mathrm{KH}_{2} \mathrm{PO}_{4} 1.0 \mathrm{~g} / \mathrm{L}$, yeast extract $5 \mathrm{~g} / \mathrm{L},\left(\mathrm{NH}_{4}\right)_{2} \mathrm{SO}_{4} 5 \mathrm{~g} / \mathrm{L}, \mathrm{NaCl} 15 \mathrm{~g} / \mathrm{L}$, glycerol $50 \mathrm{~g} / \mathrm{L}, \mathrm{MgSO}_{4} \cdot 7 \mathrm{H}_{2} \mathrm{O} 1 \mathrm{~g} / \mathrm{L}, \mathrm{CoCl}_{2} \cdot 6 \mathrm{H}_{2} \mathrm{O} 0.01 \mathrm{~g} / \mathrm{L}$, $\mathrm{MnCl}_{2} \cdot 4 \mathrm{H}_{2} \mathrm{O} 0.01 \mathrm{~g} / \mathrm{L}, \mathrm{FeSO}_{4} \cdot 7 \mathrm{H}_{2} \mathrm{O} 0.01 \mathrm{~g} / \mathrm{L}$, and vitamin $\mathrm{B}_{12} 0.005 \mathrm{~g} / \mathrm{L}$. The medium was supplemented with $100 \mu \mathrm{g} / \mathrm{mL}$ ampicillin (Amp) to keep the plasmids for the strains without a plasmid stabilization system. For the strains with a plasmid stabilization system, no antibiotics were added.
Fed-batch fermentations were carried out in T\&J MiniBox parallel bioreactors with modified VN medium at $37^{\circ} \mathrm{C}$. The $\mathrm{pH}$ was controlled at 6.5 by automatically feeding $5 \mathrm{M} \mathrm{NaOH}$. The dissolved oxygen was maintained at $10 \%$ of air saturation at an aeration rate of 1.0 vvm by adjusting the agitation speed. The feeding solution contains $600 \mathrm{~g} / \mathrm{L}$ glycerol, $10 \mathrm{~g} / \mathrm{L}$ yeast extract, $1 \mathrm{~g} / \mathrm{L} \quad \mathrm{MgSO}_{4} \cdot 7 \mathrm{H}_{2} \mathrm{O}, 0.01 \mathrm{~g} / \mathrm{L} \mathrm{CoCl}_{2} \cdot 6 \mathrm{H}_{2} \mathrm{O}, 0.01 \mathrm{~g} / \mathrm{L}$ $\mathrm{MnCl}_{2} \cdot 4 \mathrm{H}_{2} \mathrm{O}$, and $0.01 \mathrm{~g} / \mathrm{L} \mathrm{FeSO}_{4} \cdot 7 \mathrm{H}_{2} \mathrm{O}$. Another $5 \mathrm{mg} / \mathrm{L}$ vitamin $\mathrm{B}_{12}$ was added at $12 \mathrm{~h}$.

\section{Plasmid stability assay}

To determine the stability of plasmids, the culture samples were taken at different intervals during the fed-batch fermentation. The samples were diluted with water and plated for single cells on LB plates without antibiotics and incubated overnight at $37 \circ \mathrm{C}$. Colonies were replica plated onto LB plates containing $100 \mathrm{mg} / \mathrm{L}$ ampicillin to check for the presence of the plasmid. The plasmid maintenance ratio was calculated by dividing the colony numbers of LB-ampicillin plates by the colony numbers of LB plates.

\section{Quantification of biomass and products}

Cell concentration was determined by measuring the optical density of $600 \mathrm{~nm}\left(\mathrm{OD}_{600}\right)$. High-performance liquid chromatography (HPLC) equipped with an Aminex HPX-87H Column $(300 \times 7.8 \mathrm{~mm})$ and detection via UV absorption at $210 \mathrm{~nm}$ was used to determine the concentration of glycerol, 1,3-PDO, 3-HP, and other organic acids. $5 \mathrm{mM} \mathrm{H}_{2} \mathrm{SO}_{4}$ was used as the mobile phase with a flow rate of $0.8 \mathrm{~mL} / \mathrm{min}$ (Chen et al. 2014).

\section{Enzyme assays}

The activity of glycerol-3-phosphate dehydrogenase was determined by the method as described by Weiner and Heppel (1972). The activity of alcohol dehydrogenase YqhD toward 3-hydroxypropionaldehyde (3-HPA) reduction was determined as described by Pérez et al. (2008; Sulzenbacher et al. 2004). The activity of aldehyde dehydrogenase AldH toward 3-HPA oxidation was determined as described by Zhao et al. (2019). All of the measures were repeated for three times.

\section{Results and discussion}

\section{Plasmid stability assay of a recombinant $V$. natriegens during 1,3-PDO production}

In our previous work, a recombinant $V$. natriegens $\mathrm{VN} 10$ was constructed to produce 1,3-PDO from glycerol (Zhang et al. 2021). The pathways leading to the formation of main byproducts were all blocked in this strain by deleting $a d h E$ (ethanol), ldhA (lactate), $p f$ (formate), pta-ack $A$ (acetate), frdABCD (succinate), aldA, and ald $B$ 
genes (3-HP). Moreover, the $\operatorname{arcA}$ and $g l p R$ genes encoding two key transcriptional regulators (Nizam et al. 2009) and the $\operatorname{sth} A$ gene encoding a soluble pyridine nucleotide transhydrogenase were also deleted to increase NADPH availability (Fig. 1). An optimized 1,3-PDO synthesis module was overexpressed in plasmid pTrc99a by placing dhaBCE and $g d r A B$ genes encoding glycerol dehydratase and its activator under promoter $\mathrm{J} 23106$ and $y q h D$ gene encoding NADPH-dependent alcohol dehydrogenase under promoter J23100 (Fig. 1). Moreover, the phaP gene from Azotobacter sp. encoding phasin PhaP and the transhydrogenase genes $p n t A B$ were also overexpressed in the plasmid to increase the robustness of cell and the intracellular concentration of NADPH, respectively (Fig. 1). PhaP is a polyhydroxyalkanoate granule-associated protein that has been used to protect cells against several kinds of stress, including toxic metabolites, such as ethanol, butanol, and 3-hydroxybutyrate (de Almeida et al. 2007; Mezzina et al. 2017; Zhang et al. 2021). It has also been successfully used to increase the production of 1,3-PDO (Mezzina et al. 2017; Zhang et al. 2021). Strain VN10 can produce $56.4 \mathrm{~g} / \mathrm{L} 1,3-\mathrm{PDO}$ in $24 \mathrm{~h}$ with a yield of $0.61 \mathrm{~mol} / \mathrm{mol}$ glycerol during fed-batch fermentation without the accumulation of other byproducts (Fig. 2A). However, a sharp reduction of the productivity of 1,3PDO was observed after $15 \mathrm{~h}$ (Fig. 2A). Since 1,3-PDO synthesis module was overexpressed in the plasmid and the plasmid was very large $(\sim 13.7 \mathrm{~kb})$, we supposed that instability of the plasmid may be one important reason related to the reduced productivity. To test this hypothesis, we took samples at different time intervals during fed-batch fermentation and measured the plasmid maintenance ratios. As shown in Fig. 2B, a high ratio of plasmid loss was observed during fed-batch fermentation. Although antibiotic $(100 \mathrm{mg} / \mathrm{L}$ ampicillin) was added in the medium, $51.8 \%$ and $65.8 \%$ of cells lost plasmid at $12 \mathrm{~h}$ and $24 \mathrm{~h}$. We also measured the activity of NADPHdependent alcohol dehydrogenase (YqhD) during fedbatch fermentation. As shown in Fig. $2 \mathrm{C}$, the activity of YqhD was also significantly reduced after $12 \mathrm{~h}$, suggesting that the reduced productivity of 1,3-PDO may be related to plasmid loss. To reduce plasmid loss, we tried to add ampicillin at different time intervals during fed-batch fermentation, which, however, did not improve the plasmid maintenance ratios or the productivity of 1,3-PDO (data not shown).

\section{Designing a plasmid maintenance system to improve 1,3-PDO production}

To increase plasmid stability during fed-batch fermentation of $V$. natriegens, we attempted to design a plasmid maintenance system by coupling substrate consumption,

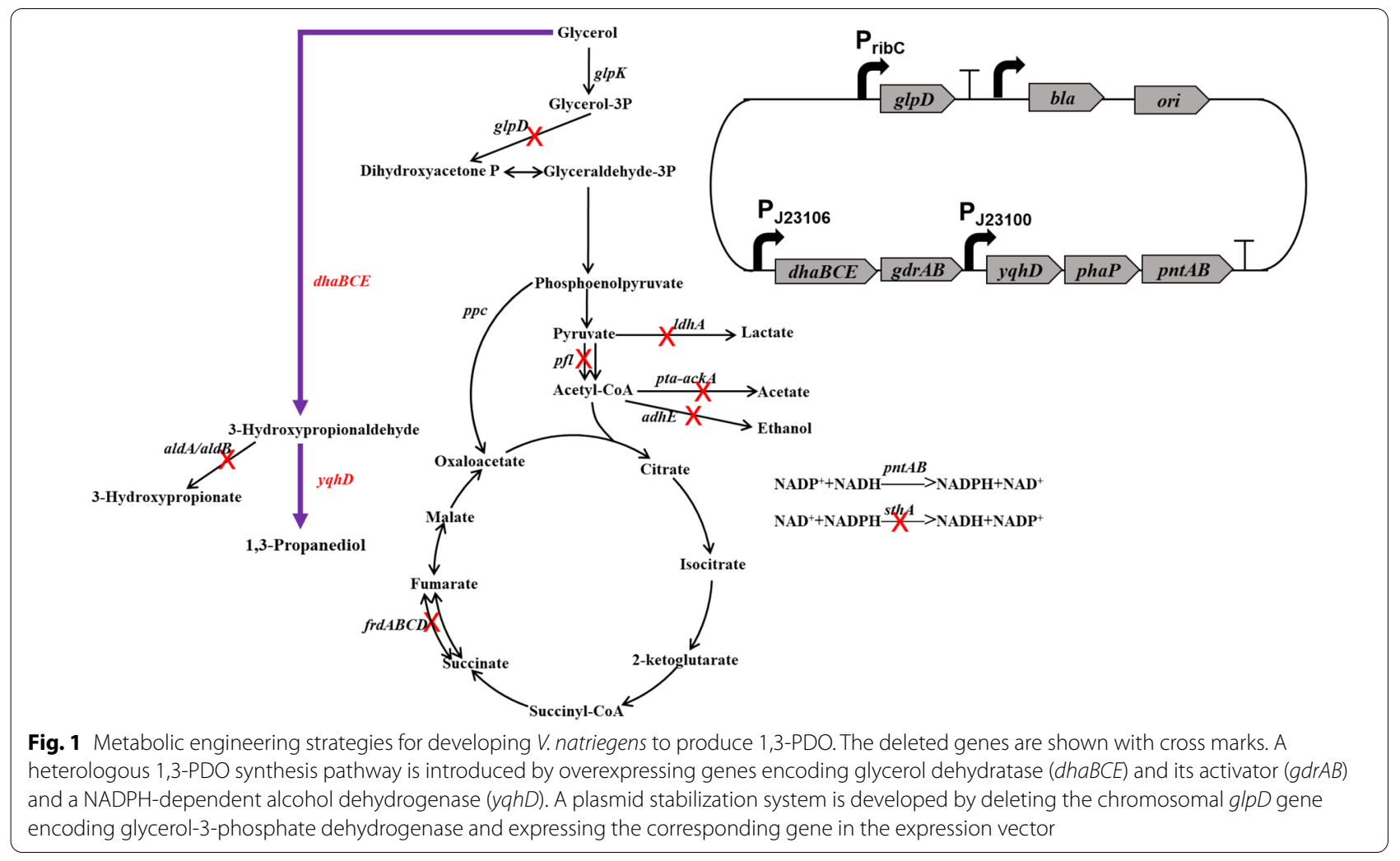



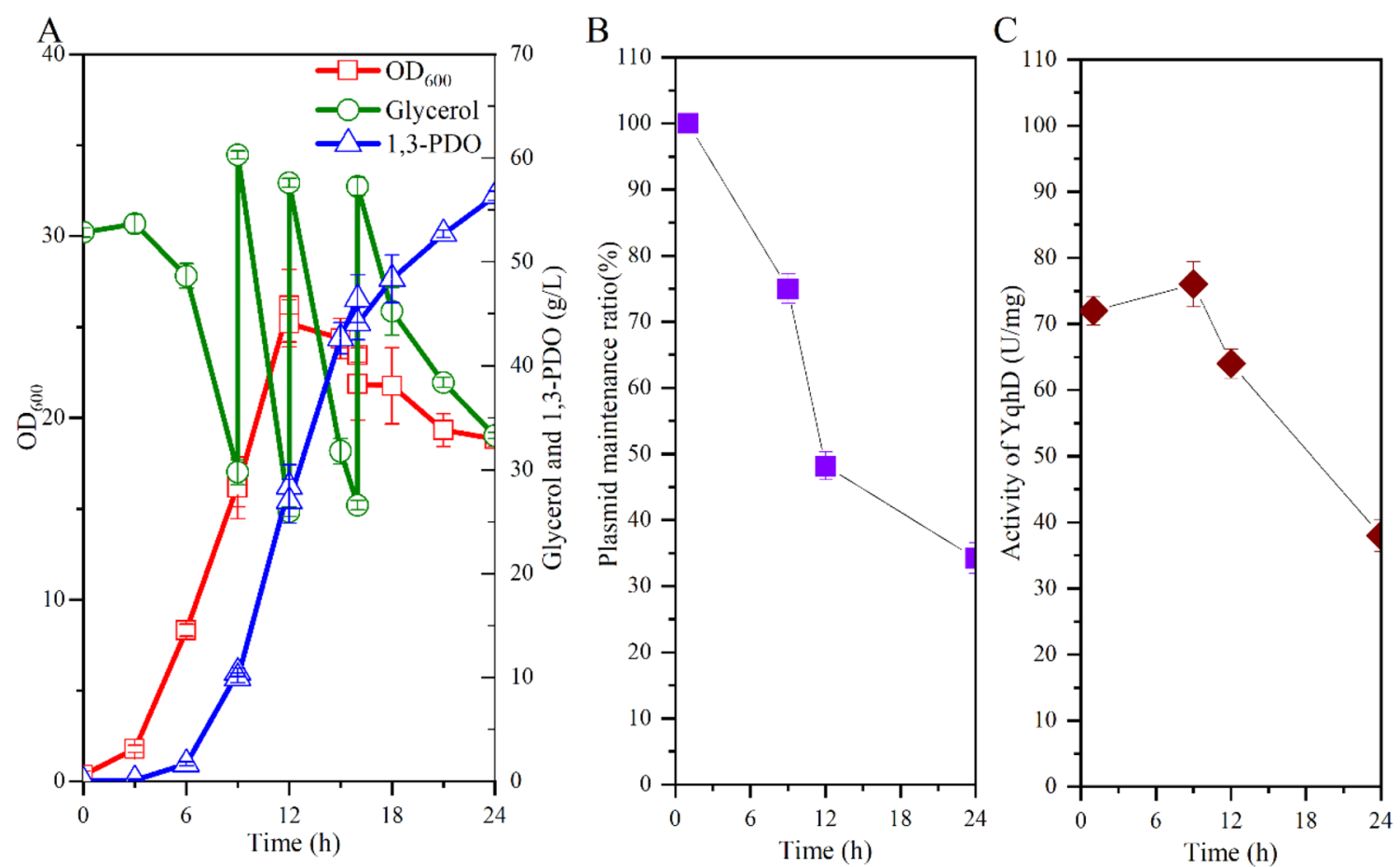

Fig. 2 Plasmid stability of V. natriegens VN10 during fed-batch fermentation. A Fermentation profiles of strain VN10; B Plasmid maintenance ratio during fed-batch cultivation; C Activity of YqhD during fed-batch cultivation. To maintain the stability of plasmid, $100 \mathrm{mg} / \mathrm{L}$ of ampicillin was added in the fermentation medium

cell viability, and product formation in the expression plasmid. $V$. natriegens has only one glycerol consumption pathway which is composed of glycerol kinase $(g l p K)$ and glycerol-3-phosphate dehydrogenase $(g l p D)$ (Zhang et al. 2021). Our previous study showed that the expression of glpK gene was subjected to complex genetic regulation (Zhang et al. 2021). To avoid significant perturbation of cellular metabolism, we select the $g l p D$ gene as a target for designing a gene complementation system. The chromosomal $g l p D$ gene was deleted and expressed in the expression plasmid pTrc99a-J23106-doy-phaP-pntAB. Knockout of $g l p D$ gene abolishes the growth of $V$. natriegens VN10 in M9 minimal medium with glycerol as the sole carbon source. $V$. natriegens $\mathrm{VN} 10 \triangle g l p D$ showed marginal growth in fermentation medium due to the presence of yeast extract but did not consume glycerol (Fig. 3A). To recover the activity of glycerol-3-phosphate dehydrogenase, the $g l p D$ gene was inserted into plasmid pTrc99a-J23106-doy-phaP-pntAB with different promoters. With the consideration of plasmid number of pTrc99a, three constitutive promoters from $V$. natriegens with varied strength $(1 \% \sim 10 \%$ of the expression level of chromosomal $g l p D$ gene) were selected, including the native promoters of $d n a G$ gene, pat $Z$ gene, and $r i b C$ gene (Lee et al. 2019; Zhang et al. 2021). As shown
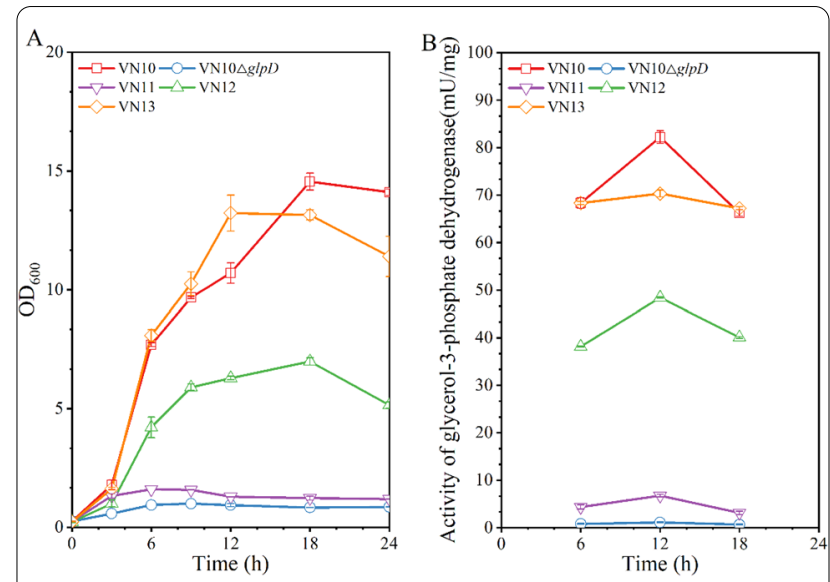

Fig. 3 Development of a plasmid maintenance system by adjusting the promoter of $g / p D$ gene in the expression vector. A Cell growth; B Activity of glycerol-3-phosphate dehydrogenase during the shake flask cultivation

in Fig. $3 \mathrm{~A}$ and $\mathrm{B}$, when the promoter of $r i b C$ gene was used to express plasmid-bearing $g l p D$ gene, strain VN13 recovered normal cell growth and showed similar activity of glycerol-3-phosphate dehydrogenase compared to strain VN10. 
The performance of strain VN13 was tested in fedbatch fermentation without adding antibiotics. As shown in Fig. 4A, strain VN13 produced 69.5 g/L 1,3-PDO in $24 \mathrm{~h}$, with a productivity of $2.90 \mathrm{~g} / \mathrm{L} / \mathrm{h}$ and a yield of $0.62 \mathrm{~mol} / \mathrm{mol}$. The productivity of 1,3-PDO was $23 \%$ higher than that obtained by strain VN10 based on the antibiotic selection system. The expression plasmid in strain VN13 was almost completely maintained during the whole fed-batch fermentation (Fig. 4B), indicating that the designed system was efficient to couple cell viability with plasmid maintenance. The activity of YqhD was also retained at a high level during the whole fedbatch fermentation (Fig. 4C), suggesting that increased plasmid stability is helpful to enhance pathway efficiency and 1,3-PDO production. The yield and productivity of 1,3-PDO by strain VN13 are higher than most natural 1,3-PDO producers (Lee et al. 2015, 2018; Sun et al. 2018;). Thus, the developed plasmid maintenance system was shown to be effective and robust for increasing 1,3PDO production under antibiotic-free cultivation, which is strongly important for large-scale fermentation in industry. Compared to strain VN10, the yield of 1,3-PDO by strain VN13 was not significantly increased probably due to that the flux distribution to glycerol oxidative and reductive pathways was partially altered due to the expression of $g l p D$ gene in the expression plasmid. It is possible to increase the yield of 1,3-PDO by further optimizing the promoter strength of $g l p D$ gene in the plasmid. The final titer of 1,3-PDO could also be increased by extending the fermentation time. However, the productivity of 1,3-PDO was significantly reduced after $24 \mathrm{~h}$ due to severe cell death (data not shown) although the cells still had a high plasmid maintenance ratio (>95\%). The reasons for the severe cell death are unknown and should be elucidated in the future.

\section{Application of the plasmid maintenance system for developing a 3-HP hyperproducer}

To test the general applicability of the designed plasmid maintenance system, we attempted to implement the system for 3-HP production. 3-HP is an important platform chemical that can be used as a precursor for the synthesis of various chemicals, such as acrylic acid, acrylonitrile, and propiolactone (Chen et al. 2017; Dishisha et al. 2015; Kim et al. 2020). Different microorganisms have been engineered to produce 3-HP, including E. coli, Klebsiella pneumoniae, and Corynebacterium glutamicum (Chen et al. 2017; Kim et al. 2020; Zhao et al. 2019). However, the productivities of 3-HP by all reported microorganisms are lower than $2.5 \mathrm{~g} / \mathrm{L} / \mathrm{h}$, which can be a limiting
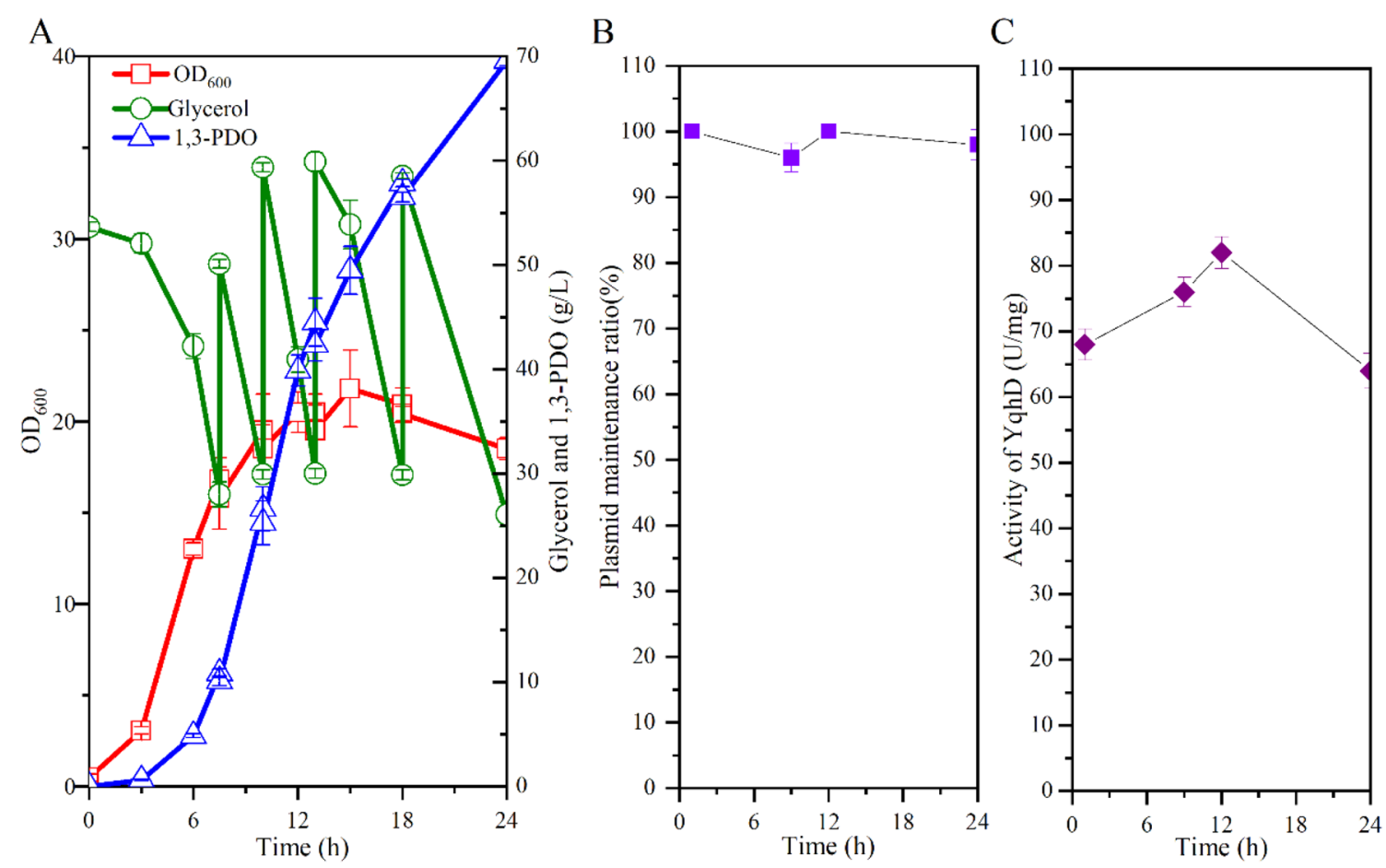

Fig. 4 Increasing the production of 1,3-PDO by strain VN13 with a plasmid stabilization system in fed-batch fermentation. A Fermentation profiles of strain VN13; B Plasmid maintenance ratio during fed-batch cultivation; C Activity of YqhD during fed-batch cultivation. No antibiotics were added in the fermentation medium 
factor for its industrial application. Considering its fast growth and high substrate consumption rate, $V$. natriegens could be a potential chassis for 3-HP production. Since the conversion of glycerol to 3-HP is an oxidative process which generates $\mathrm{NADH}$, we constructed a new $V$. natriegens starting strain VN05 by deleting the $a d h E$, $l d h A$, pfl, pta-ackA, and frdABCD genes to prevent the accumulate of other byproducts (Fig. 5). The 3-HP synthesis module was firstly established by constructing plasmid pTrc99a-dhaBCE-aldH which overexpressed $d h a B C E$ and $g d r A B$ genes under promoter J23106 and ald $H$ gene encoding an efficient aldehyde dehydrogenase from E. coli under promoter J23100 (Jo et al. 2008). The phasin PhaP gene phaP was also introduced to increase the strain's tolerance to 3-HP (de Almeida et al. 2007; Mezzina et al. 2017). By introducing plasmid pTrc99adhaBCE-aldH into $V$. natriegens $\mathrm{VN} 05$, the generated strain VN14 was able to accumulate $49.5 \mathrm{~g} / \mathrm{L} 3-\mathrm{HP}$ by fed-batch fermentation with the addition of ampicillin, with a productivity of $2.06 \mathrm{~g} / \mathrm{L} / \mathrm{h}$ and a yield of $0.68 \mathrm{~mol} /$ mol (Fig. 6A). No other byproducts were detected during the fermentation. However, severe loss of plasmid was also observed during the fed-batch fermentation (Fig. 6B). At $24 \mathrm{~h}$, the plasmid maintenance ratio was only
$32 \%$ and the activity of aldehyde dehydrogenase was also sharply reduced after $9 \mathrm{~h}$ (Fig. 6B and C). Thus, the plasmid maintenance system was also introduced by deleting the chromosomal $g l p D$ gene and expressing it in plasmid pTrc99a-dhaBCE-aldH-glpD3 under the control of ribC promoter. The resulting strain VN15 produced $64.5 \mathrm{~g} / \mathrm{L}$ 3-HP during the fed-batch fermentation without adding antibiotics, with a productivity of $2.69 \mathrm{~g} / \mathrm{L} / \mathrm{h}$ and a yield of $0.67 \mathrm{~mol} / \mathrm{mol}$ (Fig. 6D). The expression plasmid was almost completely maintained and the activity of aldehyde dehydrogenase was retained at a high level during the fermentation (Fig. $6 \mathrm{E}$ and F). Thus, the developed plasmid maintenance system was also effective for increasing 3-HP production under antibiotic-free cultivation. To the best of our knowledge, the obtained productivity of 3-HP was the highest value reported to date.

\section{Conclusion}

In this study, a plasmid maintenance system was developed to increase the stability of large plasmids in $V$. natriegens. By deleting the chromosomal $g l p D$ gene and complementing it in an expression vector, the system was shown to be effective to prevent plasmid loss since the $\operatorname{glpD}$ gene is essential for cell survival and glycerol

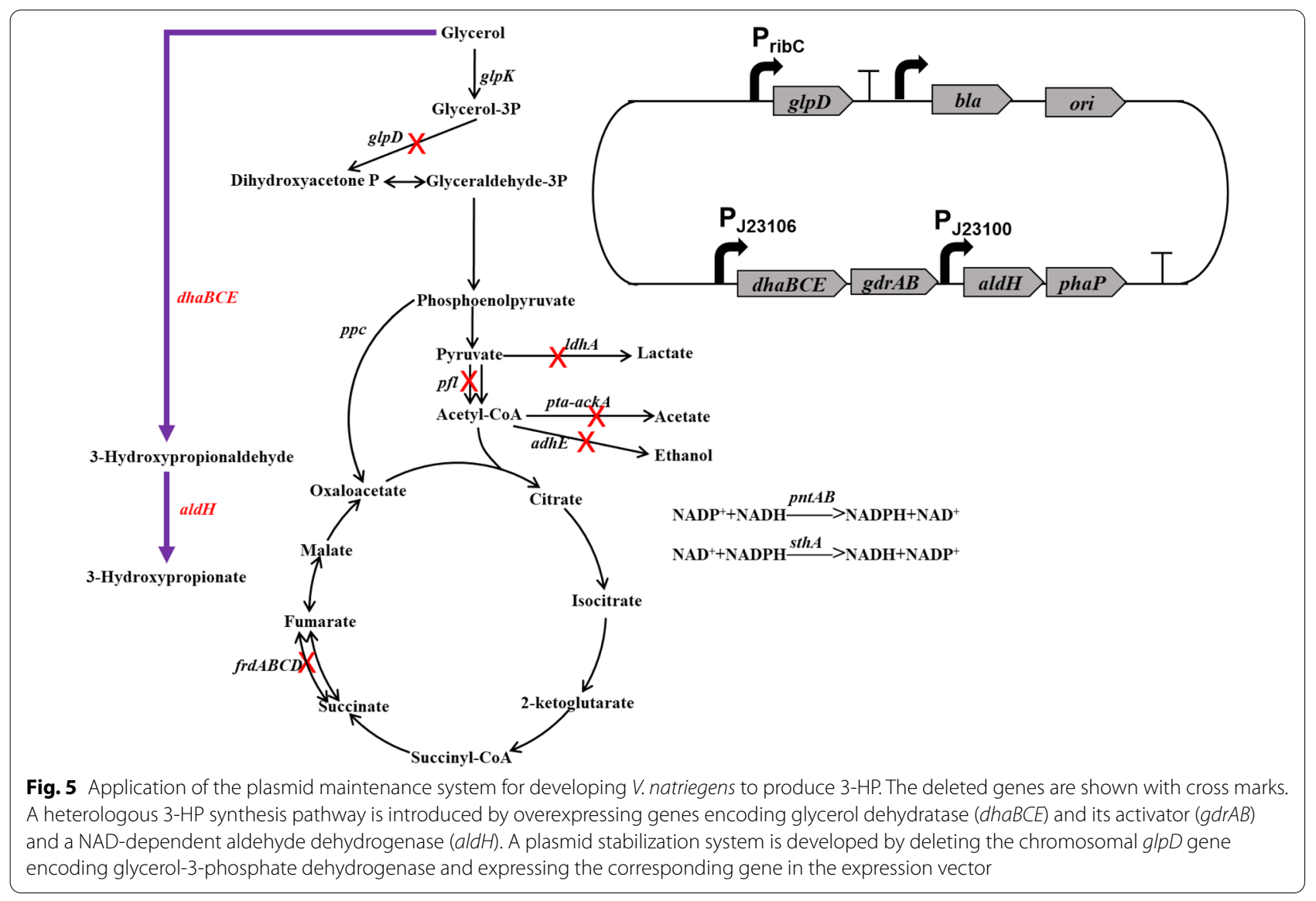



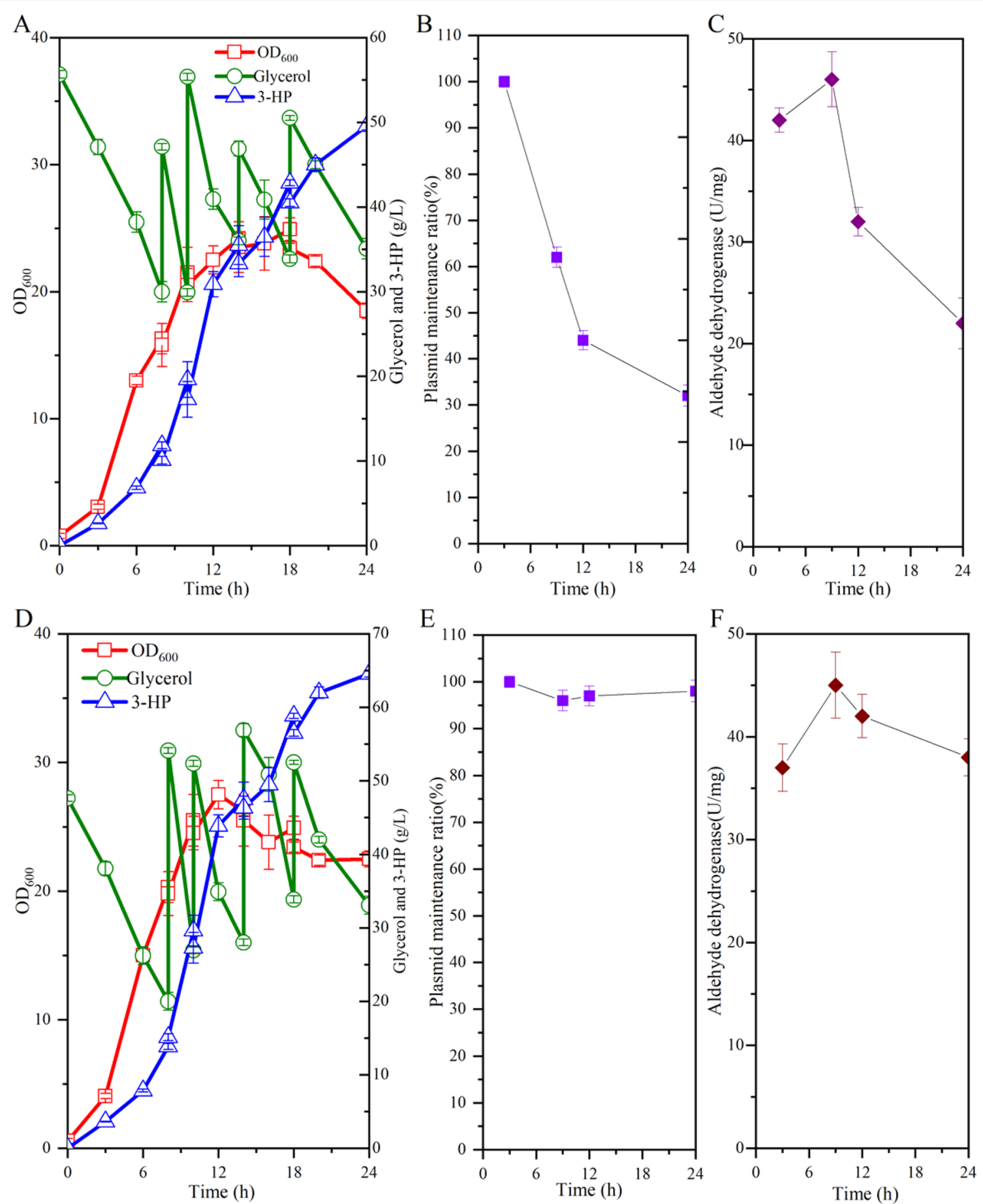

Fig. 6 Production of 3-HP by strain VN14 and strain VN15 (with a plasmid maintenance system) during fed-batch fermentation. A Fermentation profiles of strain VN14; B Plasmid maintenance ratio by strain VN14; C Activity of aldehyde dehydrogenase of strain VN14; D Fermentation profiles of strain VN15; E Plasmid maintenance ratio by strain VN15; F Activity of aldehyde dehydrogenase of strain VN15. 100 mg/L of ampicillin was added during the fermentation of strain VN14, while no antibiotics were added during the fermentation of strain VN15

consumption in the cultivation using glycerol as the sole carbon source. The system was successfully implemented to engineer $V$. natriegens to produce 1,3-PDO and 3-HP from glycerol, enabling the resulting strains to produce $69.5 \mathrm{~g} / \mathrm{L} 1,3-\mathrm{PDO}$ and $64.5 \mathrm{~g} / \mathrm{L} 3-\mathrm{HP}$ in $24 \mathrm{~h}$. The obtained productivities were significantly higher than 
those by antibiotic selection cultivations. The developed antibiotic-free system provided an important tool for engineering $V$. natriegens as an industrial chassis for the production of chemicals on a large scale. The system can also be modified to produce chemicals from other carbon sources by selecting alternative essential genes.

\section{Abbreviations}

1,3-PDO: 1,3-Propanediol; 3-HP: 3-Hydroxypropionate; $\mathrm{OD}_{600}$ : Optical density at wavelength $600 \mathrm{~nm}$; HPLC: High-performance liquid chromatography.

\section{Supplementary Information}

The online version contains supplementary material available at https://doi. org/10.1186/s40643-021-00485-0.

Additional file 1: Table S1. Primers used in this study.

\section{Acknowledgements}

This work was supported by the National Key R\&D Program of China (No. 2019YFE0196900), the National Natural Science Foundation of China (Grant Nos. 21878172, 21938004, and 22078172), and the DongGuan Innovative Research Team Program (No. 201536000100033).

\section{Authors' contributions}

$Z C$ and $D L$ designed the research. $Y Z$ and $Z C$ wrote the paper. $Y Z$ analyzed the data. $Y Z, Y S, Y L$, and $X C$ performed the research. ZC supervised the paper and has funding acquisition. All the authors read and approved the final manuscript.

\section{Funding}

This work was supported by the National Key R\&D Program of China (No. 2019YFE0196900), the National Natural Science Foundation of China (Grant Nos. 21878172, 21938004, and 22078172), and the DongGuan Innovative Research Team Program (No. 201536000100033).

\section{Availability of data and materials}

Not applicable.

\section{Declarations}

Ethics approval and consent to participate

Not applicable.

\section{Consent for publication}

All the authors consented to the publication of this work.

\section{Competing interests}

The authors declare that they have no competing interests.

\section{Author details}

${ }^{1}$ Key Laboratory of Industrial Biocatalysis (Ministry of Education), Department of Chemical Engineering, Tsinghua University, Beijing 100084, China. ${ }^{2}$ Tsinghua Innovation Center in Dongguan, Dongguan 523808, China. ${ }^{3}$ Center for Synthetic and Systems Biology, Tsinghua University, Beijing 100084, China.

Received: 29 October 2021 Accepted: 7 December 2021 Published online: 14 December 2021

\section{References}

Becker J, Wittmann C (2015) Advanced biotechnology: metabolically engineered cells for the bio-based production of chemicals and fuels, materials, and health-care products. Angew Chem Int Ed Engl 54:3328-3350
Chen Z, Bommareddy RR, Frank D, Rappert S, Zeng AP (2014) Deregulation of feedback inhibition of phosphoenolpyruvate carboxylase for improved lysine production in Corynebacterium glutamicum. Appl Environ Microbiol 80:1388-1393

Chen Z, Huang J, Wu Y, Wu W, Zhang Y, Liu D (2017) Metabolic engineering of Corynebacterium glutamicum for the production of 3-hydroxypropionic acid from glucose and xylose. Metab Eng 39:151-158

Clomburg JM, Crumbley AM, Gonzalez R (2017) Industrial biomanufacturing: the future of chemical production. Science 355:804-814

Dalia AB, McDonough E, Camilli A (2014) Multiplex genome editing by natural transformation. Proc Natl Acad Sci USA 111:8937-8942

Dalia TN, Hayes CA, Stolyar S, Marx CJ, McKinlay JB, Dalia AB (2017) Multiplex genome editing by natural transformation (MUGENT) for synthetic biology in Vibrio natriegens. ACS Synth Biol 6:1650-1655

de Almeida A, Nikel PI, Giordano AM, Pettinari MJ (2007) Effects of granuleassociated protein PhaP on glycerol-dependent growth and polymer production in poly(3-hydroxybutyrate)-producing Escherichia coli. Appl Environ Microbiol 73:7912-7916

Dishisha T, Pyo SH, Hatti-Kaul R (2015) Bio-based 3-hydroxypropionic- and acrylic acid production from biodiesel glycerol via integrated microbial and chemical catalysis. Microb Cell Fact 14:200

Hoffart E, Grenz S, Lange J, Nitschel R, Muller F, Schwentner A, Feith A, LenfersLucker M, Takors R, Blombach B (2017) High substrate uptake rates empower Vibrio natriegens as production host for industrial biotechnology. Appl Environ Microbiol 83:e01614-e1617

Jo JE, Mohan Raj S, Rathnasingh C, Selvakumar E, Jung WC, Park S (2008) Cloning, expression, and characterization of an aldehyde dehydrogenase from Escherichia coli K-12 that utilizes 3-Hydroxypropionaldehyde as a substrate. Appl Microbiol Biotechnol 81:51-60

Kang WK, Lim HG, Yang J, Noh MH, Seo SW, Gung GY (2018) Synthetic auxotrophs for stable and tunable maintenance of plasmid copy number. Metab Eng 48:121-128

Kim JW, Ko Y-S, Chae TU, Lee SY (2020) High-level production of 3-hydroxypropionic acid from glycerol as a sole carbon source using metabolically engineered Escherichia coli. Biotechnol Bioeng 117(7):2139-2152

Ko Y-S, Kim JW, Lee JA, Han T, Kim GB, Park JE, Lee SY (2020) Tools and strategies of systems metabolic engineering for the development of microbial cell factories for chemical production. Chem Soc Rev 49:4615-4636

Lee CS, Aroua MK, Daud WMAW, Cognet P, Pérès-Lucchese Y, Fabre PL, Reynes O, Latapie L (2015) A review: Conversion of bioglycerol into 1,3-propanediol via biological and chemical method. Renew Sust Energ Rev 42:963-972

Lee JH, Jung MY, Oh MK (2018) High-yield production of 1,3-propanediol from glycerol by metabolically engineered Klebsiella pneumoniae. Biotechnol Biofuels 11:104

Lee HH, Ostrov N, Wong BG, Gold MA, Khalil AS, Church GM (2019) Functional genomics of the rapidly replicating bacterium Vibrio natriegens by CRISPRi. Nat Microbiol 4:1105-1113

Lim HG, Kwak DH, Park S, Woo S, Yang JS, Kang CW, Kim B, Noh MH, Seo SW, Jung GY (2019) Vibrio sp. dhg as a platform for the biorefinery of brown macroalgae. Nat Commun 10:2486

Mezzina MP, Alvarez DS, Egoburo DE, Diaz Pena R, Nikel PI, Pettinari MJ (2017) A new player in the biorefineries field: phasin PhaP enhances tolerance to solvents and boosts ethanol and 1,3-propanediol synthesis in Escherichia coli. Appl Environ Microbiol 83:e00662-e717

Nizam SA, Zhu J, Ho PY, Shimizu K (2009) Effects of $\operatorname{arcA}$ and $\operatorname{arcB}$ genes knockout on the metabolism in Escherichia coli under aerobic condition. Biochem Eng J 44:240-250

Pérez JM, Arenas FA, Pradenas GA, Sandoval JM, Vásquez CC (2008) Escherichia coli YahD exhibits aldehyde reductase activity and protects from the harmful effect of lipid peroxidation-derived aldehydes. J Biol Chem 283(12):7346-7353

Rugbjerg P, Sommer M (2019) Overcoming genetic heterogeneity in industrial fermentations. Nat Biotechnol 37:869-876

Sulzenbacher G, Alvarez K, Van Den Heuvel RH, Versluis C, Spinelli S, Campanacci V, Valencia C, Cambillau C, Eklund H, Tegoni M (2004) Crystal structure of E.coli alcohol dehydrogenase YqhD: evidence of a covalently modified NADP coenzyme. J Mol Biol 342:489-502

Sun YQ, Shen JT, Yan L, Zhou JJ, Jiang LL, Chen Y, Yuan JL, Feng E, Xiu ZL (2018) Advances in bioconversion of glycerol to 1,3-propanediol: prospects and challenges. Process Biochem 71:134-146 
Terrinoni M, Nordqvist S, Källgård S, Holmgren J, Lebensa M (2018) A novel nonantibiotic, lgt-based selection system for stable maintenance of expression vectors in Escherichia coli and Vibrio cholerae. Appl Environ Microbiol 84:e02143-17.

Wang Z, Tschirhart T, Schultzhaus Z, Kelly EE, Chen A, Oh E, Nag O, Glaser E, Kim E, Lloyd P, Charles P, Li W, Leary D, Compton J, Phillips D, Dhinojwala A, Payne G, Vora G (2020) Melanin produced by the fast-growing marine bacterium Vibrio natriegens through heterologous biosynthesis: characterization and application. Appl Environ Microbiol 86:e02749-e2719

Weiner JH, Heppel LA (1972) Purification of the membrane-bound and pyridine nucleotide-independent L-glycerol 3-phosphate dehydrogenase from Escherichia coli. Biochem Biophys Res Commun 47:1360-1365

Weinstock MT, Hesek ED, Wilson CM, Gibson DG (2016) Vibrio natriegens as a fast-growing host for molecular biology. Nat Methods 13:849-851

Zhang Y, Liu D, Chen Z (2017) Production of C2-C4 diols from renewable bioresources: new metabolic pathways and metabolic engineering strategies. Biotechnol Biofuels 10:299

Zhang Y, Li Z, Liu Y, Cen X, Liu D, Chen Z (2021) Systems metabolic engineering of Vibrio natriegens for the production of 1,3-propanediol. Metab Eng 65:52-65

Zhao P, Ma C, Xu L, Tian P (2019) Exploiting tandem repetitive promoters for high-level production of 3-hydroxypropionic acid. Appl Microbiol Biotechnol 103:4017-4031

\section{Publisher's Note}

Springer Nature remains neutral with regard to jurisdictional claims in published maps and institutional affiliations.

\section{Submit your manuscript to a SpringerOpen ${ }^{\odot}$ journal and benefit from:}

- Convenient online submission

- Rigorous peer review

- Open access: articles freely available online

- High visibility within the field

- Retaining the copyright to your article

Submit your next manuscript at $\gg$ springeropen.com 